\title{
Indirect link between resonant and guided modes on uniform and periodic slabs
}

\author{
Amgad Abdrabou* and Ya Yan Lu \\ Department of Mathematics, City University of Hong Kong, Kowloon, Hong Kong, China
}

(Dated: January 28, 2019)

\begin{abstract}
A uniform or periodic dielectric slab can serve as an optical waveguide for which guided modes are important, and it can also be used as a diffraction structure for which resonant modes with complex frequencies are relevant. Guided modes are normally studied below the lightline where they exist continuously and emerge from points on the lightline, but isolated guided modes may exist above the lightline and they are the so-called bound states in the continuum. Resonant modes are usually studied above the lightline (defined using the real part of the complex frequency), but they are not connected to the guided modes on the lightline. In this work, through analytic and numerical calculations for uniform and periodic slabs, we establish an indirect link between the resonant and guided modes. It is shown that as the (Bloch) wavenumber is increased, a resonant mode continues its existence below the lightline, until it reaches its end at an Exceptional Point (EP) where a pair of improper modes emerge, and one branch of improper modes eventually approaches the lightline at the starting point of a guided mode. Leaky modes with a real frequency and a complex (Bloch) wavenumber (propagation constant) are also related to the improper modes. They emerge at EPs in eigenvalue formulations where the frequency is regarded as a parameter. Our study is based on a non-Hermitian eigenvalue formulation that includes resonant, improper and leaky modes, and provides a complete picture for different kinds of eigenmodes on uniform and periodic slabs.
\end{abstract}

PACS numbers: 42.65.Hw,42.25.Fx,42.79.Dj

\section{INTRODUCTION}

Eigenvalue problems for photonic structures such as optical waveguides, resonant cavities and periodic media are of fundamental importance 1, 2]. Although the research topic has a long history, new and interesting wave phenomena related to eigenvalue problems are continuously being discovered. Recently, bound states in the continuum (BICs) [3], which are trapped or guided modes with their frequencies within the radiation continuum, have attracted much attention and found a number of significant applications in optics 4 6]. Exceptional points (EPs) [7, 8], which are spectral degeneracies for nonHermitian eigenvalue problems with coalescing eigenvalues and eigenfunctions, are being intensively investigated in optics [9 12], and may have valuable applications in lasing and sensing [13, 14]. EPs are also the key to many unusual and counter-intuitive wave phenomena in $\mathcal{P} \mathcal{T}$ symmetric and related structures 15 18]. For dielectric structures that are unbounded in one or two spatial directions, the most important eigenvalue problems are those for guided and resonant modes [19]. Guided modes decay exponentially in the media surrounding the structure, and usually exist below the lightline. The resonant modes radiate power to infinity, have complex frequencies, and are usually studied above the lightline. The BICs are isolated guided modes above the lightline, and they can also be regarded as special resonant modes with infinite quality factors [5]. EPs of resonant modes have been found on various open dielectric structures $9-13$. This is not unexpected, since the eigenvalue problems for res-

* Corresponding author: mabdrabou2-c@my.cityu.edu.hk onant modes on open structures are non-Hermitian due to radiation losses.

For unbounded structures that are periodic or invariant in one or two spatial directions, band structures for guided and resonant modes have been calculated by many authors. Below the lightline, there are continuous branches of guided modes, and they emerge from starting points on the lightline. Resonant modes are often calculated above the lightline, and they seem to approach the lightline near the starting points of the guided modes. However, careful numerical studies show that the guided modes and the resonant modes are not connected on the lightline. In fact, the resonant modes cross the lightline with a nonzero imaginary part in their complex frequencies. We believe it is interesting to study the resonant modes below the lightline, and establish a link between the resonant and guided modes. To the best of our knowledge, such a link has never been established before. To find the missing link between these two kinds of modes, we concentrate on simple uniform or periodic dielectric slabs in this paper. Our study relies on a mathematical formulation that includes not only the resonant modes, but also improper modes that grow exponentially in the surrounding media and leaky modes with real frequencies and complex wavenumbers [1]. Our analysis reveals that as the wavenumber is increased, a typical branch of resonance modes continues its existence below the lightline until it reaches an EP where a pair of improper modes emerge, and one branch of the improper modes eventually ends up on the lightline where it connects to the guided modes.

The rest of this paper is organized as follows. In Sec. II, we present some basic properties for eigenvalue problems on one-dimensional (1D) structures for which a uniform slab is a special case. In Sec. III, we show numerical 
results for different types of eigenmodes on a slab with a constant refractive index. Section IV is devoted to a periodic slab with a piecewise constant refractive index profile. The paper is concluded by some remarks and discussions in Sec. V.

\section{EIGENMODES ON 1D STRUCTURES}

We consider a 1D dielectric wave-guiding structure surrounded by air. A Cartesian coordinate system is chosen so that the structure is perpendicular to the $z$ axis. The relative permittivity depends only on $z$ and satisfies $\varepsilon(z)=1$ for $|z|>b$ and $\varepsilon(z)=\varepsilon_{1}(z)$ for $|z|<b$, where $\varepsilon_{1}(z) \geq 1, \max \left[\varepsilon_{1}(z)\right]>1$, and $h=2 b$ is the thickness of the structure. A uniform slab, shown in Fig. 1 below, is

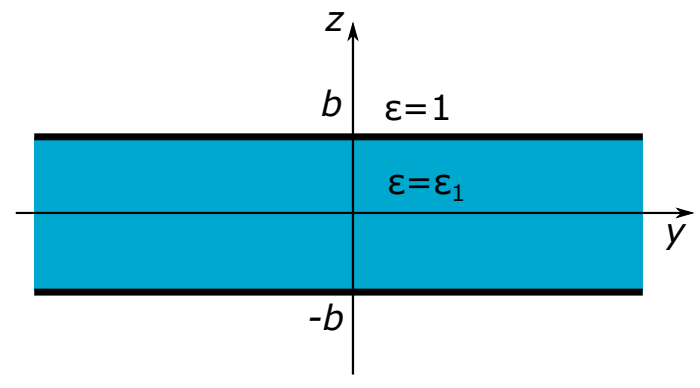

FIG. 1: A uniform slab of thickness $h=2 b$ and relative permittivity $\varepsilon_{1}$ surrounded by air.

a special case for which $\varepsilon_{1}(z)$ is a constant.

Assuming the field is invariant in the $x$ direction, a transverse electric (TE) eigenmode of the structure is a solution with its electric field component $E_{x}$ given as

$$
E_{x}=\operatorname{Re}\left[\phi(z) e^{i(\beta y-\omega t)}\right]
$$

where $\phi(z)$ is the mode profile, $\beta$ is the propagation constant, $\omega$ is the angular frequency, $k=\omega / c$ is the free space wavenumber, and $c$ is the speed of light in vacuum. The function $\phi$ satisfies the following 1D Helmholtz equation

$$
\frac{d^{2} \phi}{d z^{2}}+k^{2} \varepsilon(z) \phi=\beta^{2} \phi
$$

for $-\infty<z<\infty$, subject to boundary conditions that determine the physical nature of the solution.

Guided modes are solutions for real $\beta$ and real $k$, such that $\phi(z) \rightarrow 0$ as $z \rightarrow \pm \infty$. For $|z|>b$, Eq. (2) is reduced to $d^{2} \phi / d z^{2}+\left(k^{2}-\beta^{2}\right) \phi=0$, and the solutions are either oscillatory or exponential depending on whether $k^{2}-\beta^{2}$ is positive or negative. Therefore, guided modes only exist when $k<|\beta|$, that is, below the lightline. Moreover, the guided modes satisfy the following boundary condition

$$
\frac{d \phi}{d z}=\mp \gamma_{0} \phi \quad \text { at } \quad z= \pm b
$$

where $\gamma_{0}=\sqrt{\beta^{2}-k^{2}}$. Equation (2) for $|z|<b$ and Eq. (3) give rise to an eigenvalue problem of the guided modes on the finite interval $(-b, b)$ for $z$. Assuming $\beta$ is given, then $k$ or $k^{2}$ can be regarded as the eigenvalue. Alternatively, if $k$ is given, then $\beta$ or $\beta^{2}$ is the eigenvalue. Since $\gamma_{0}$ depends on the eigenvalue, the above formulation on the finite interval of $z$ is nonlinear.

For a guided mode $\{\beta, k, \phi(z)\}$, the derivative of $k$ with respect to $\beta$ is given by

$$
\frac{d k}{d \beta}=\frac{\beta \int_{-\infty}^{\infty}|\phi(z)|^{2} d z}{k \int_{-\infty}^{\infty} \epsilon(z)|\phi(z)|^{2} d z} .
$$

The above can be derived by taking the partial derivative of Eq. (2) with respect to $\beta$, multiplying the resulting equation by $\bar{\phi}$ (the complex conjugate of $\phi$ ) and integrating with respect to $z$ on $(-\infty, \infty)$. It is clear that for $\beta>0, d k / d \beta>0$, thus, the dispersion curves of guided modes always have a positive slope. Equation (4) can be written as

$$
\frac{d k}{d \beta}=\frac{\beta}{k} \cdot \frac{2 \gamma_{0} \int_{-b}^{b}|\phi(z)|^{2} d z+|\phi(b)|^{2}+|\phi(-b)|^{2}}{2 \gamma_{0} \int_{-b}^{b} \epsilon(z)|\phi(z)|^{2} d z+|\phi(b)|^{2}+|\phi(-b)|^{2}}
$$

Therefore, as the dispersion curve approaches the lightline for $\beta>0$, we have $\beta / k \rightarrow 1, \gamma_{0} \rightarrow 0$ and $d k / d \beta \rightarrow 1$. In other words, at the limiting points on the lightline, the dispersion curves of guided modes are tangential to the lightline.

For real $\beta$ and real $k<|\beta|$, Eq. (2) also has solutions that blow up at infinity, i.e., $\phi(z) \rightarrow \infty$ as $z \rightarrow \pm \infty$. These solutions have been referred to as improper modes 20, 21], and they satisfy the boundary condition

$$
\frac{d \phi}{d z}= \pm \gamma_{0} \phi \quad \text { at } \quad z= \pm b
$$

The improper modes are unphysical when they are considered on the infinite interval of $z$, but they have physical meaning on any bounded interval $(-D, D)$ for $D>b$. If we put proper waveguides with high refractive indices in $z>D$ and $z<-D$, respectively, a supermode of the multilayered structure may have the same $\beta$ and same $k$ as the improper mode, and the mode profile of the supermode is then identical to that of the improper mode for $z \in(-D, D)$.

Resonant modes are solutions of the Helmholtz equation for real $\beta$ and complex $k$ (i.e., complex $\omega$ ) that exhibit outgoing wave behavior as $z \rightarrow \pm \infty$. They radiate power to infinity and decay with time. Under the time dependence given in Eq. (1), we have $\operatorname{Im}(\omega)<0$. If we define $\alpha_{0}=\sqrt{k^{2}-\beta^{2}}$ based on the standard complex square root with the real negative axis as the branch cut, then $\phi(z)=\phi(b) e^{i \alpha_{0}(z-b)}$ for $z>b$ and $\phi(z)=\phi(-b) e^{-i \alpha_{0}(z+b)}$ for $z<-b$. Therefore, the resonant modes satisfy the following boundary conditions

$$
\frac{d \phi}{d z}= \pm i \alpha_{0} \phi \quad \text { at } \quad z= \pm b .
$$

Multiplying Eq. (2) by $\bar{\phi}$, integrating the result on 
$(-b, b)$, and applying the boundary condition (6), we obtain

$$
\begin{aligned}
i \alpha_{0} & {\left[|\phi(b)|^{2}+|\phi(-b)|^{2}\right] } \\
& =\int_{-b}^{b}\left|\frac{d \phi}{d z}\right|^{2} d z+\int_{-d}^{d}\left[\beta^{2}-k^{2} \varepsilon_{1}(z)\right]|\phi|^{2} d z .
\end{aligned}
$$

The imaginary part of above gives

$$
\operatorname{Im}(k)=\frac{-\operatorname{Re}\left(\alpha_{0}\right)\left[|\phi(b)|^{2}+|\phi(-b)|^{2}\right]}{2 \operatorname{Re}(k) \int_{-b}^{b} \varepsilon_{1}(z)|\phi(z)|^{2} d z} .
$$

If $k$ is complex, then $\operatorname{Re}\left(\alpha_{0}\right)>0$ due to the definition of the square root, and Eq. (8) gives $\operatorname{Im}(k)<0$. This is consistent with the requirement that the resonant modes should decay with time. In addition, we have $\operatorname{Im}\left(\alpha_{0}\right)<0$, thus $\phi$ blows up as $z \rightarrow \pm \infty$. The resonant modes are usually studied above the lightline, that is for $\operatorname{Re}(k)>$ $|\beta|$. In general, as $\operatorname{Re}(k) \rightarrow \beta, \operatorname{Im}(k)$ and $\alpha_{0}$ do not tend to 0 , thus, the resonant modes and guided modes are not connected on the lightline. In latter sections, we show that the resonant modes continue their existence below the lightline.

If $\{k, \phi\}$ is a resonant mode for a real $\beta$, then $\{\bar{k}, \bar{\phi}\}$ is another solution of Eq. (2) for the same $\beta$. If we denote that solution also by $\{k, \phi\}$, then it satisfies

$$
\frac{d \phi}{d z}=\mp i \alpha_{0} \phi \quad \text { at } \quad z= \pm b .
$$

For the time dependence given in Eq. (11), a solution satisfying Eqs. (2) and (9) represents the time reversal of a resonant mode. The field consists of incoming waves from $z= \pm \infty$ and its amplitude grows with time. On the other hand, if we replace the the time dependence by $e^{i \omega t}$, then a resonant mode satisfies Eq. (9) and its time reversal satisfies Eq. (6). We point out that both the resonant modes and their time reversals satisfy boundary condition (5), with $\gamma_{0}=\sqrt{\beta^{2}-k^{2}}$ defined using the standard complex square root. This is so, because for a resonant mode, $\operatorname{Im}(k)<0$, thus $\operatorname{Im}\left(\beta^{2}-k^{2}\right)>0, \operatorname{Im}\left(\gamma_{0}\right)>0$, and $\gamma_{0}=i \alpha_{0}$; and for the time reversal of the resonant mode, $\operatorname{Im}(k)>0$, thus $\operatorname{Im}\left(\beta^{2}-k^{2}\right)<0, \operatorname{Im}\left(\gamma_{0}\right)<0$, and $\gamma_{0}=-i \alpha_{0}$. Therefore, the boundary condition (5) allows us to unify the improper modes, the resonant modes and their time reversals. Actually, this is quite natural, since all these modes blow up as $z \rightarrow \pm \infty$.

For optical waveguides, leaky modes with real frequencies and complex propagation constants are widely studied [1]. Under the dependence on $t$ and $y$ given in Eq. (1), the mode profile satisfies the same Helmholtz equation (2). Notice that a leaky mode with $\operatorname{Re}(\beta)>0$ should have $\operatorname{Im}(\beta)>0$, so that as it propagates forward in the positive $y$ direction, it radiates power to infinity (for $z \rightarrow \pm \infty)$, and its amplitude decreases. If $\{\beta, \phi\}$ is a leaky mode, then its complex conjugate $\{\bar{\beta}, \bar{\phi}\}$ represents a time reversed solution that increases as it propagates forward by gaining power coming from infinity (i.e., from $z= \pm \infty)$. Alternatively, $\{\bar{\beta}, \bar{\phi}\}$ can also be regarded as a leaky mode with the dependence on $t$ and $y$ switched to $e^{i(\omega t-\beta y)}$. Once again, the boundary condition (5) is applicable to both leaky modes and their time reversals.

In summary, a 1D dielectric wave-guiding structure supports guided and improper modes for real $\beta$ and real $k$, resonant modes and their time reversals for real $\beta$ and complex $k$, leaky modes and their time reversals for real real $k$ and complex $\beta$. Except for the guided modes, all other modes blow up as $z \rightarrow \pm \infty$ and satisfy boundary condition (5).

\section{UNIFORM SLAB: NUMERICAL RESULTS}

In this section, we present numerical results for a uniform slab with a constant refractive index, that is, $\varepsilon(z)=\varepsilon_{1}$, a constant, for $-b<z<b$. Due to reflection symmetry with respect to the mid-plane of the slab, the eigenmodes are either even in $z$ or odd in $z$. For the even and odd modes, the mode profile can normalized by the condition $\phi(b)=1$, and be written as

$$
\phi(z)=\frac{\cos \left(\gamma_{1} z\right)}{\cos \left(\gamma_{1} b\right)} \quad \text { or } \quad \phi(z)=\frac{\sin \left(\gamma_{1} z\right)}{\sin \left(\gamma_{1} b\right)}
$$

for $|z|<b$, respectively, where $\gamma_{1}=\sqrt{k^{2} \varepsilon_{1}-\beta^{2}}$.

For the guided modes, we have $\phi(z)=e^{-\gamma_{0}(z-b)}$ for $z>b$, then the continuity of $d \phi / d z$ at $z=b$ gives us the following equation

$$
e^{2 i \gamma_{1} b} \mp \frac{i \gamma_{0}+\gamma_{1}}{i \gamma_{0}-\gamma_{1}}=0,
$$

where the + and - signs correspond to the even and odd modes, respectively. The guided modes form discrete branches below the lightline, and $m$-th dispersion curve approaches the lightline $(k=\beta)$ at

$$
k=\beta=k_{m}=\frac{\pi P_{m}}{d \sqrt{\varepsilon_{1}-1}}
$$

where $P_{m}=m$ for the even modes and $P_{m}=m-1 / 2$ for the odd modes, and $m \geq 1$.

For the improper, resonant and leaky modes, and their time reversals, we need to use boundary condition (5), and thus $\phi(z)=e^{\gamma_{0}(z-b)}$ for $z>b$. From the continuity of $d \phi / d z$ at $z=b$, we obtain

$$
e^{2 i \gamma_{1} b} \mp \frac{\gamma_{0}+i \gamma_{1}}{\gamma_{0}-i \gamma_{1}}=0 .
$$

Again, the + and - signs correspond to the even and odd modes, respectively.

To find the dispersion relations of various modes, we only have to solve Eqs. (10) and (12). As a numerical example, we consider a slab with $\varepsilon_{1}=11.56$. In Figs. 2a and 2b we show the dispersion curves of the first four 


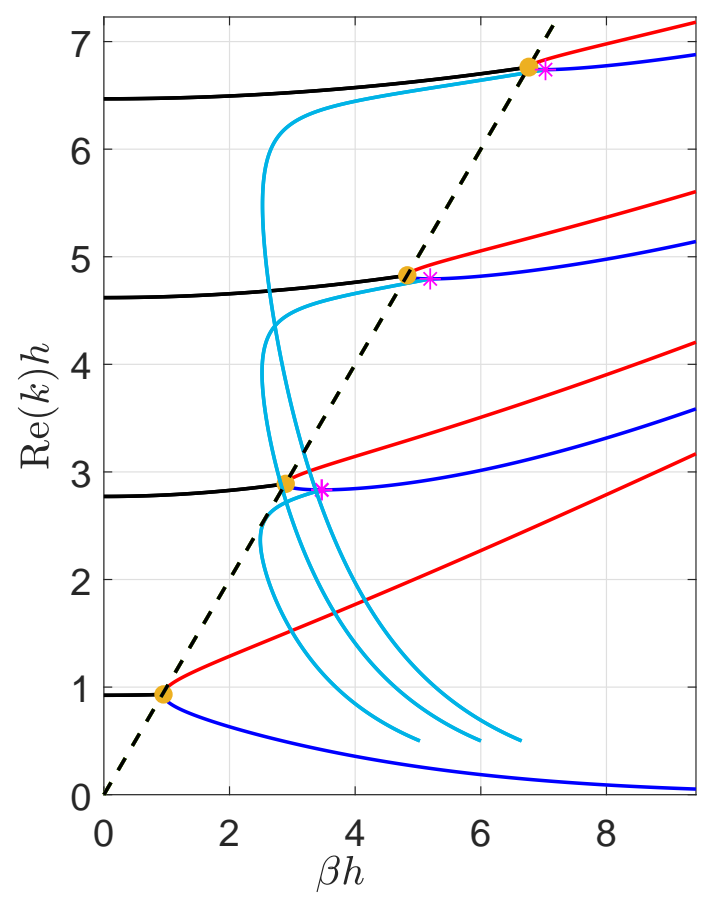

(a)

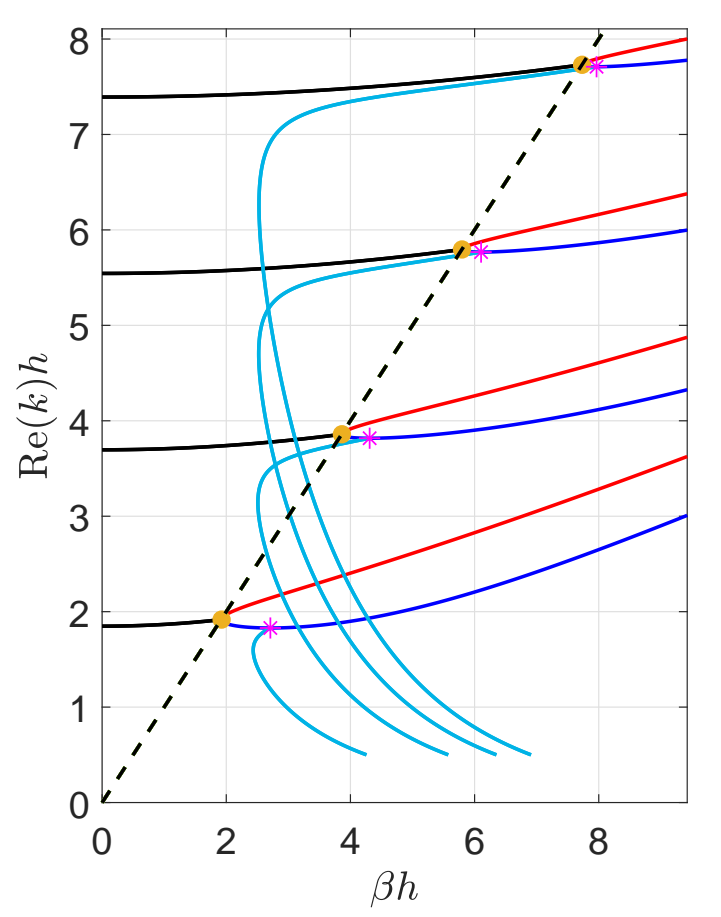

(b)

FIG. 2: Dispersion curves of (a) odd and (b) even modes for $1 \leq m \leq 4$, showing guided (red), resonant (black), improper (blue) and leaky (cyan) modes. odd and even modes, respectively. In particular, we show $\operatorname{Re}(k) h$ for the resonant modes and $\operatorname{Re}(\beta) h$ for the leaky modes. The guided, resonant, leaky and improper modes are shown in red, black, cyan and blue, respectively. Notice that there is no odd leaky mode for $m=1$.

From Fig. 2, we observe that the resonant modes approach the lightline near the starting points of the guided modes, but actually these two kind of modes are not connected on the lightline. In Fig. 3, we show the first
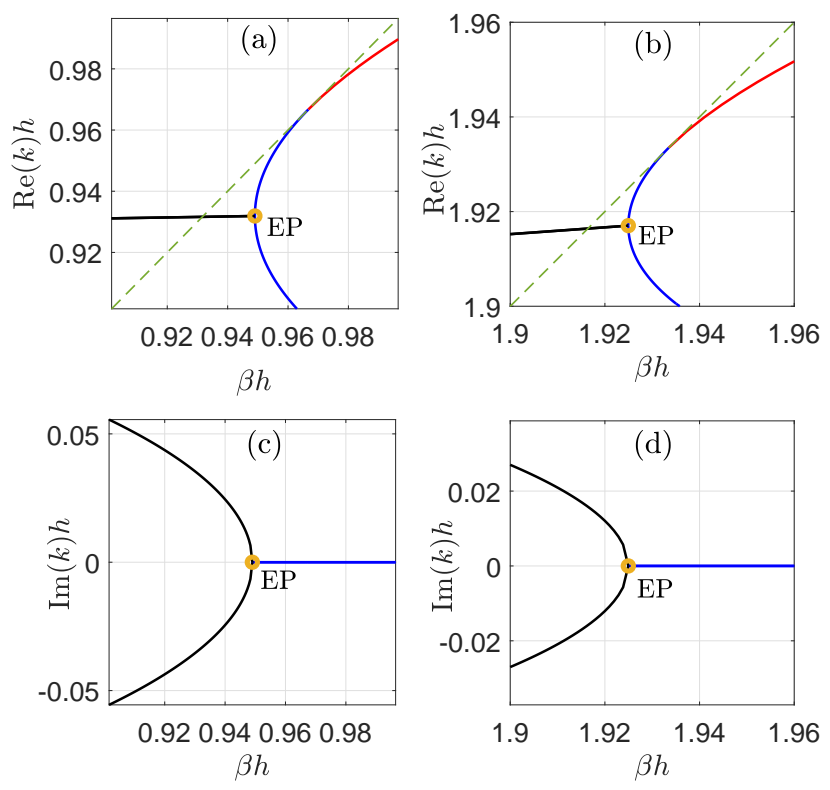

FIG. 3: Real parts (Figs. 3a and 3b) and imaginary parts (Figs. 3 . and $3 \mathrm{~d}$ ) of $k h$ versus $\beta h$ for the odd and even modes with $m=1$, respectively.

odd and even modes near the lightline. The red, black and blue curves correspond to guided, resonant and improper modes, respectively. The guided modes converge to the lightline tangentially, confirming the theoretical result given in Sec. II. For the resonant modes (and their time reversals), we show $\operatorname{Re}(k h)$ in Figs. 3 and $3 \mathrm{~b}$, and $\operatorname{Im}(k h)$ in Figs. 3 r and $3 \mathrm{~d}$. Figures $3 \mathrm{Ba}$ and 3 r show the first odd mode, and Figs. 3b and 3 $\mathrm{d}$ correspond to the first even mode. Notice that the resonant mode and its time reversal exist below the lightline until they reach an EP, beyond which a pair of improper modes emerge. More precisely, as $\beta$ increases through the EP, a complex conjugate pair of $k$ (for a resonant mode and its time reversal) is turned to two different real values of $k$ corresponding to two improper modes. In addition, $\operatorname{Im}(k)$ and $\operatorname{Re}(k)$ exhibit a one-sided square root splitting before and after the EP, respectively. When $\beta$ is further increased, one branch of the improper modes converge to the lightline at exactly the same point where a branch of guided modes emerges. The higher order modes (with $m>1$ ) have exactly the same behavior. 
The EPs separating resonant and improper modes can be easily calculated, since they correspond to $d k / d \beta=$ $\infty$. From Eq. (12) and its derivative, we obtain the following system

$$
\begin{aligned}
& \gamma_{1}+\varepsilon_{1} \gamma_{0} \cot \left(\gamma_{1} b\right)-\varepsilon_{1} b \gamma_{1} \gamma_{0} \csc ^{2}\left(\gamma_{1} b\right)=0 \\
& \gamma_{1} \cot \left(\gamma_{1} b\right)-\gamma_{0}=0
\end{aligned}
$$

for EPs of odd modes, and

$$
\begin{aligned}
& \gamma_{1}-\varepsilon_{1} \gamma_{0} \tan \left(\gamma_{1} b\right)-\varepsilon_{1} b \gamma_{1} \gamma_{0} \sec ^{2}\left(\gamma_{1} b\right)=0 \\
& \gamma_{1} \tan \left(\gamma_{1} b\right)+\gamma_{0}=0
\end{aligned}
$$

for EPs of even modes. The above systems can be easily solved. The results are listed in the following table.

\begin{tabular}{c|c|c|c}
\hline Parity & $m$ & $\beta h$ & $k h$ \\
\hline odd & 1 & 0.9490247327 & 0.9319093681 \\
\hline odd & 2 & 2.8945994396 & 2.8893869146 \\
\hline odd & 3 & 4.8303964776 & 4.8272891351 \\
\hline odd & 4 & 6.7648851107 & 6.7626694968 \\
\hline even & 1 & 1.9249524220 & 1.9170319451 \\
\hline even & 2 & 3.8627853763 & 3.8588932776 \\
\hline even & 3 & 5.7977220843 & 5.7951354860 \\
\hline even & 4 & 7.7319467271 & 7.7300088986 \\
\hline
\end{tabular}

TABLE I: Exceptional points in the $\beta-k$ plane separating resonant modes and improper modes.

Leaky modes with real $k$ and complex $\beta$ are also shown in Fig. 2. It is known that the leaky modes are connected to the improper modes [20, 21]. In fact, leaky modes emerge from local minima of the dispersion curves of the improper modes. For the uniform slab considered in this section, there is no leaky mode associated with the lowest odd improper mode $(m=1)$, since for that mode, $k$ decreases monotonically as $\beta$ is increased. In Figs. 4 a and 4 , we show the dispersion curves, $k h$ versus $\operatorname{Re}(\beta) h$, for the second odd mode $(m=2)$ and the first even mode $(m=1)$. The imaginary parts of $\beta h$ are shown in Figs. 40 and $4 \mathrm{~d}$. The red, blue and magenta curves correspond to the guided, improper and leaky modes, respectively. It is clear that as $k$ is decreased, two real $\beta$ (for two improper modes) are turned to a complex conjugate pair of $\beta$ through an EP. The complex conjugate pair of $\beta$ represents a leaky mode and its time reversal.

The EPs separating the leaky and improper modes can also be easily calculated, since they satisfy the necessary condition $d k / d \beta=0$. From Eq. (12) and its derivative, we obtain the following system

$$
\begin{aligned}
& \gamma_{1}+\gamma_{0} \cot \left(\gamma_{1} b\right)-b \gamma_{1} \gamma_{0} \csc ^{2}\left(\gamma_{1} b\right)=0 \\
& \gamma_{1} \cot \left(\gamma_{1} b\right)-\gamma_{0}=0
\end{aligned}
$$

for EPs of the odd modes, and

$$
\begin{aligned}
& \gamma_{1}-\gamma_{0} \tan \left(\gamma_{1} b\right)-b \gamma_{1} \gamma_{0} \sec ^{2}\left(\gamma_{1} b\right)=0 \\
& \gamma_{1} \tan \left(\gamma_{1} b\right)+\gamma_{0}=0
\end{aligned}
$$

for EPs of the even modes.
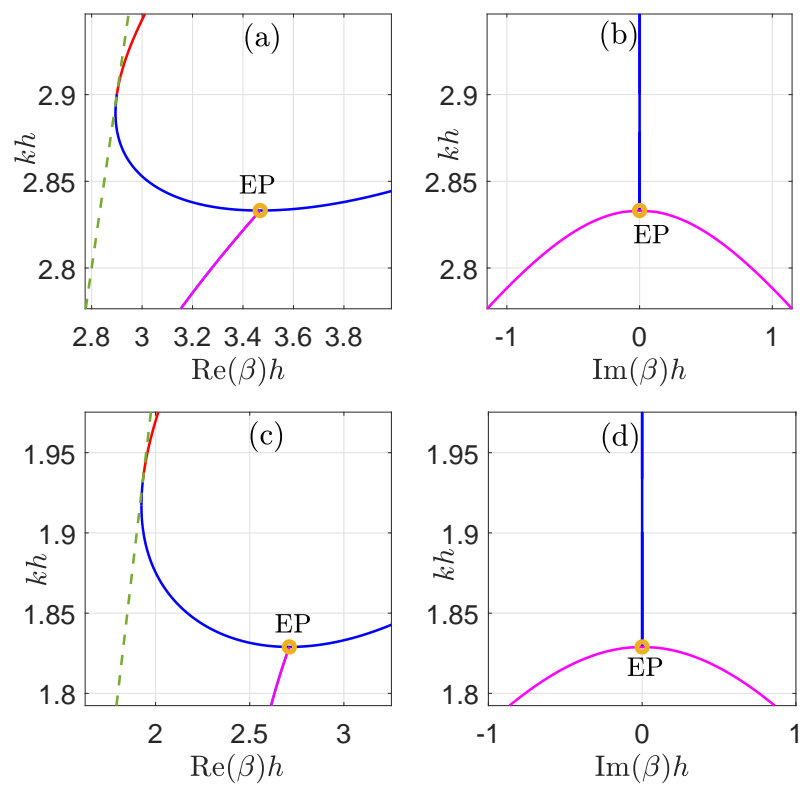

FIG. 4: Normalized free-space wavenumber $k h$ versus $\operatorname{Re}(\beta) h$ (Figs. 4h and $4 \mathrm{c})$ and $\operatorname{Im}(\beta) h($ Figs. $4 \mathrm{~b}$ and $4 \mathrm{~d})$ for the leaky odd $(m=2)$ and even $(m=1)$ modes, respectively.

\section{PERIODIC SLAB}

In this section, we consider a simple two-dimensional (2D) dielectric structure, that is, a periodic slab with two uniform segments, and show that the resonant and guided modes of the periodic slab are also linked under the lightline through EPs and improper modes. A schematic of the periodic slab is shown in Fig. 5 , where $z$

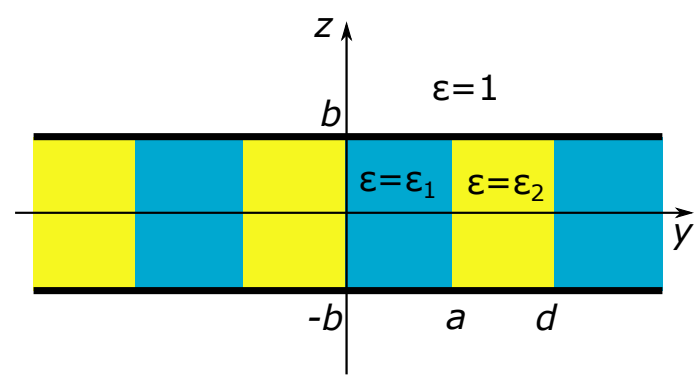

FIG. 5: A dielectric slab that is invariant in $x$ and periodic in $y$ with period $d$. Each period consists of two uniform segments.

is perpendicular to the slab, the $x y$ plane is the mid-plane of the slab, $h=2 b$ is the slab thickness, $d$ is the period in the $y$ direction, $a$ and $d-a$ are the widths of the two uniform segments with dielectric constants $\varepsilon_{1}$ and $\varepsilon_{2}$, respectively. The slab is invariant in the $x$ direction and surrounded by air (for $z>b$ and $z<-b$ ). 
Similar to Sec. II, we give definitions and boundary conditions for various modes on the periodic slab. For the $E$-polarization, the $x$ component of the electric field is assumed to be $E_{x}=\operatorname{Re}\left(u e^{-i \omega t}\right)$, where $u=u(y, z)$ satisfies the following 2D Helmholtz equation

$$
\partial_{y}^{2} u+\partial_{z}^{2} u+k^{2} \varepsilon(y, z) u=0,
$$

and $\varepsilon$ is the dielectric function. A Bloch mode of the periodic slab is a solution of Eq. (17) given in the form

$$
u(y, z)=\phi(y, z) e^{i \beta y},
$$

where $\beta$ is the Bloch wavenumber and $\phi(y, z)$ is periodic in $y$ with period $d$. Thus, $u$ is quasi-periodic in $y$ satisfying

$$
u(y+d, z)=e^{i \beta d} u(y, z) .
$$

On a lossless dielectric slab, a guided Bloch mode has a real $\beta$ and a real $k$ (i.e., a real frequency), and decays exponentially away from the slab (i.e., as $z \rightarrow \pm \infty$ ). Due to the periodicity in $y$, it is only necessary to consider $\beta$ in the interval $[-\pi / d, \pi / d]$. Below the lightline (i.e., for $k<|\beta|$ ), guided modes exist continuously with respect to $\beta$ or $k$. For $|z|>b$, the wave field of a guided mode (below the lightline) can be expanded in evanescent plane waves as

$$
u(y, z)=\sum_{m=-\infty}^{\infty} \hat{u}_{m}^{ \pm} e^{i \beta_{m} y \mp \gamma_{m} z}, \quad \pm z>b,
$$

where $\hat{u}_{m}^{ \pm}$are expansion coefficients, and

$$
\beta_{m}=\beta+2 \pi m / d, \quad \gamma_{m}=\sqrt{\beta_{m}^{2}-k^{2}} .
$$

Notice that $\beta_{0}=\beta$, all $\gamma_{m}$ are positive, $\gamma_{0}$ is identical to that defined in Sec. II, but $\gamma_{1}$ is different from that of Sec. III. If we define a linear operator $\Lambda=\Lambda(\beta, k)$, such that

$$
\Lambda e^{i \beta_{m} y}=-\gamma_{m} e^{i \beta_{m} y}, \quad m=0, \pm 1, \pm 2, \ldots
$$

then the guide mode satisfies the following boundary condition

$$
\partial_{z} u= \pm \Lambda u, \quad z= \pm b .
$$

A Bloch resonant mode on any $2 \mathrm{D}$ open periodic structure is a solution that radiates power to infinity and decays with time. It is given in Eq. (18) for a real $\beta$ and a complex $k$ (or $\omega$ ) with $\operatorname{Im}(k)<0$. Resonant modes are usually studied above the lightline, i.e., for $\operatorname{Re}(k)>|\beta|$. For $|z|>b$, the wave field of a resonant mode can also be expanded, but one or more terms in Eq (20) must be replaced by outgoing waves with growing amplitudes. For simplicity, we concentrate on resonant modes with only one outgoing wave component. In that case, the wave field can be written as

$$
\begin{aligned}
u(y, z) & =\hat{u}_{0}^{ \pm} e^{i \beta_{0} y \pm \gamma_{0} z} \\
& +\sum_{m \neq 0} \hat{u}_{m}^{ \pm} e^{i \beta_{m} y \mp \gamma_{m} z}, \quad \pm z>b .
\end{aligned}
$$

Notice that the sign for $\gamma_{0} z$ is switched. In the above, $\beta_{m}$ and $\gamma_{m}$ are still given in Eq. (21), but all $\gamma_{m}$ are complex and they are defined using the standard complex square root with a branch cut on the negative real axis. Since $k$ has a negative imaginary part, $\beta^{2}-k^{2}$ is in the upper complex half-plane, thus $\gamma_{0}$ is in the first quadrant. Since $\operatorname{Im}\left(\gamma_{0}\right)>0$, the term $e^{\gamma_{0} z}$ represents a plane wave propagating towards $z=+\infty$. Since $\operatorname{Re}\left(\gamma_{0}\right)$ is also positive, the amplitude of that plane wave grows exponentially as $z \rightarrow+\infty$. If we define a linear operator $\Lambda_{0}=\Lambda_{0}(\beta, k)$ satisfying Eq. (22) for all $m \neq 0$ and

$$
\Lambda_{0} e^{i \beta_{0} y}=\gamma_{0} e^{i \beta_{0} y},
$$

then the resonant mode satisfies the boundary condition

$$
\partial_{z} u= \pm \Lambda_{0} u, \quad z= \pm b .
$$

We emphasize that Eqs. (24) and (26) are valid for resonant modes with only one radiation channel (i.e. the plane wave for $m=0$ ) in each side of the periodic slab.

If $\{u, \beta, k\}$ is a resonant mode, then $\bar{u}$ (the complex conjugate of $u$ ) satisfies Helmholtz equation (17) with $k$ replaced by $\bar{k}$. If $\bar{u}$ is considered with the original time dependence, that is, assuming $E_{x}=\operatorname{Re}\left(\bar{u} e^{-i \omega t}\right)$, then it represents a solution that grows with time. From the complex conjugate of Eq. (24), we see that $\bar{u}$ contains the term $e^{-i \beta y+\bar{\gamma}_{0} z}$ for $z>b$, and it represents an incoming wave from $z=+\infty$. Therefore, $\bar{u}$ represents the timereversal of a resonant mode, it gains power from incoming waves and grows with time. However, $\bar{u}$ does not satisfy boundary condition (26) with $\Lambda_{0}=\Lambda_{0}(\beta, \bar{k})$, because it has a quasi-periodicity in $y$ corresponding to $-\beta$ (instead of $\beta)$. Nevertheless, corresponding to the pair $(\beta, k)$ of a resonant mode $u$, there is another resonant mode $v$ with Bloch wavenumber $-\beta$ and the same $k$. The complex conjugate of $v$ satisfies Eq. (26) with $\Lambda_{0}=\Lambda_{0}(\beta, \bar{k})$. Therefore, when Eq. (17) is solved with boundary conditions (19) and (26), we should obtain complex conjugate pairs $k$ and $\bar{k}$. Assuming $\operatorname{Im}(k)<0$, then the solution $u$ corresponding to $k$ is a resonant mode that decays with time, and the solution $\bar{v}$ corresponding to $\bar{k}$ can be regarded as the time-reversal of a resonant mode $v$ which has the same complex frequency as $u$ but propagates in the opposite direction in $y$.

Like the the uniform slab, the periodic slab also has improper modes (for real $k$ and real $\beta$ ) that grow exponentially as $z \rightarrow \pm \infty$. Since the wave field can be expanded in plane waves for $|z|>b$, there are different improper modes depending on how many of these terms are exponentially increasing. For an improper Bloch mode under the lightline with only one exponentially increasing term for $z \rightarrow+\infty$ and $z \rightarrow-\infty$, respectively, the expansion (24) is also valid. Therefore, these improper modes satisfy boundary condition (26).

For the periodic slab, Eq. (17) also has solutions with real $k$ (i.e. real frequency) and complex $\beta$. A leaky Bloch mode is a solution that radiates power to the surrounding homogeneous media (i.e. to $z= \pm \infty$ ) and decreases 
with $y$ as it propagates forward. If a leaky mode propagates in the positive $y$ direction, then the imaginary part of its complex Bloch wavenumber $\beta$ must be positive. Assuming the leaky mode has only one radiation channel corresponding to $m=0$, we claim that it also satisfies Eq. (24) and boundary condition (26). For such a leaky mode, we have $\operatorname{Im}\left(\gamma_{0}\right)>0$, thus the plane wave $e^{i \beta y+\gamma_{0} z}$ radiates power to $z=+\infty$. Similar to the case for resonant modes, when a leaky mode is found by solving Eq. (17) together with boundary conditions (19) and (26), we also obtain a solution with $\bar{\beta}$. If that solution is also considered with the time dependence $e^{-i \omega t}$, it represents the time-reversal of a leaky modes, namely, it gains power from infinity and grows as it propagates forward.

Using a highly accurate numerical method [12], we calculate guided, resonant, improper and leaky modes for a periodic slab. In Fig. 6, we show the first two odd modes (odd in $z$ ) for a periodic slab with $\varepsilon_{1}=11.56, \varepsilon_{2}=1$, $a=0.5 d$ and $h=d$. The guided, resonant and improper modes are shown in red, black and blue, respectively. In the left panel, only the real part of $k$ is shown for the resonant modes. The middle and right panels show details around the starting points of the guided modes on the lightline. It is clear that the resonant modes are not directly connected to the guided modes. Instead, as the Bloch wavenumber $\beta$ is increased, the resonant modes continue their existence under the lightline (i.e., for $\operatorname{Re}(k)<|\beta|)$, end at EPs where pairs of improper modes emerge, and the upper branches of the improper modes approach the lightline at exactly the same points where the guided modes emerge. Two lower panels in Fig. [6] show the imaginary parts of $k$ and $\bar{k}$, corresponding to the resonant modes and their time-reversals. Therefore, although the resonant and guided modes are not directly connected, they are indirectly linked via EPs and improper modes. This appears to be true for both uniform and periodic slabs.

From the results of Sec. III, we expect the leaky modes to emerge from local minima of the dispersion curves of improper modes. Since the two improper modes shown in Fig. 6] do not have local minima for $\beta \in[0, \pi / d]$, we turn to a periodic slab with a different parameter. In the left panel of Fig. 7, we show two even modes (even in $y$ ) for a periodic slab with thickness $h=3 d$ and the same $\varepsilon_{1}, \varepsilon_{2}$ and $a$. The red, black, blue and cyan curves correspond to the guided, resonant, improper and leaky modes, respectively. It is clear that the lower improper mode (with smaller $k$ ) has a local minimum, and a leaky mode and its time-reversal appear as $k$ is decreased. The minimum point is an EP for the non-Hermitian eigenvalue problem formulated using Eq. (24) or Eq. (26), and it occurs at $k=0.1323(2 \pi / d)$. The middle and right panels of Fig. 7 show more details around the EP. The real and imaginary parts of $\beta$ and $\bar{\beta}$ are shown in the middle and right panels, respectively. As we have discussed earlier, a leaky mode is associated with a complex $\beta$ with positive imaginary part, and its time-reversal is associated with $\bar{\beta}$.

\section{CONCLUSION}

A uniform or periodic dielectric slab works as an optical waveguide or a diffraction structure, depending on whether light is propagating in or illuminated on the slab. For waveguides, it is important to study guided modes, as well as leaky modes with complex propagation constants. For diffraction structures, resonant modes are highly relevant for various applications. Resonant modes are usually studied above the lightline, they approach the lightline with a nonzero imaginary part in their complex frequencies, and are not connected with the guided modes on the lightline. In this paper, using a more inclusive eigenvalue formulation and highly accurate numerical methods, we found an indirect link between the resonant and guided modes for uniform and periodic slabs. Such a link exists below the lightline, and contains an EP and improper modes. We also show that the leaky modes are related to the improper modes and emerge from EPs when frequency is regarded as the parameter.

Many existing studies on resonant and guided modes use eigenvalue formulations that are supposed to be valid for both guided and resonant modes. Numerical studies often use perfectly matched layers (PMLs) [22, 23] that force resonant modes (with outgoing wave components) to decay in the surrounding homogeneous media, and be compatible with the guided modes. Studies based on these formulations cannot find the indirect link, because they are incompatible with the improper modes. We have used one formulation for the guided modes, and another formulation for all modes that blow up at infinity. The second formulation is valid for resonant, leaky and improper modes, and the time-reversals of the resonant and leaky modes, and it allows us to identify the branching points between resonant (or leaky) and improper modes as EPs. Our study provides a complete picture for eigenmode structures on uniform and periodic slabs. We expect the main conclusions remain valid for more complicated structures, including biperiodic structures such as photonic crystal slabs.

\section{ACKNOWLEDGMENTS}

The second author acknowledges support from the Research Grants Council of Hong Kong Special Administrative Region, China (Grant No. CityU 11304117).
[1] D. Marcuse, Theory of Dielectric Optical Waveguides, 2nd ed. (Academic Press, Boston, 1991).
[2] J. D. Joannopoulos, S. G. Johnson, J. N. Winn, and R. D. Meade, Photonic Crystals: Molding the Flow of 

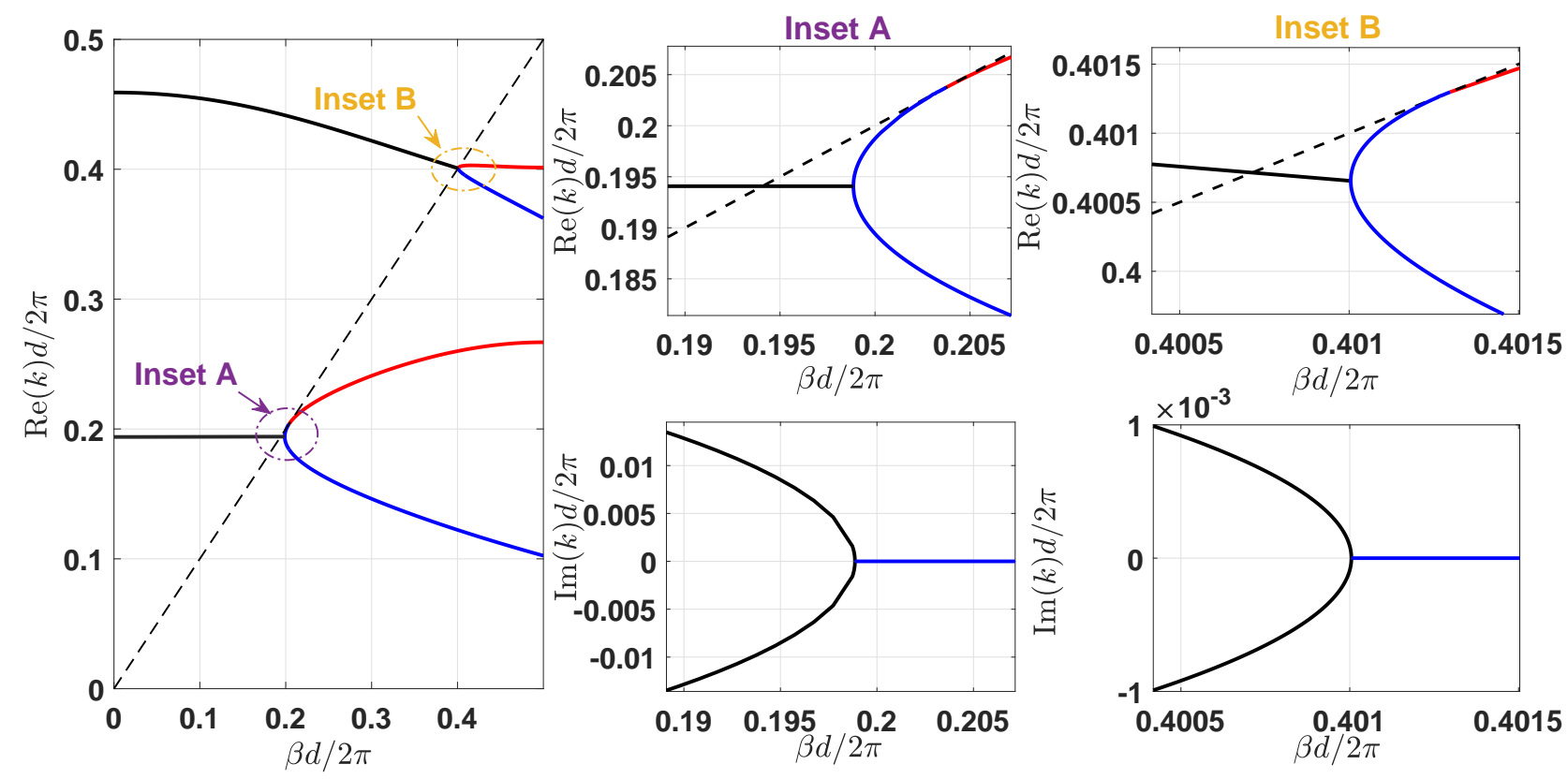

FIG. 6: Dispersion curves of the first two odd modes for a periodic slab with $\varepsilon_{1}=11.56, \varepsilon_{2}=1, a=0.5 d$ and $h=d$.

The guided, resonant and improper modes are shown in red, black and blue, respectively.
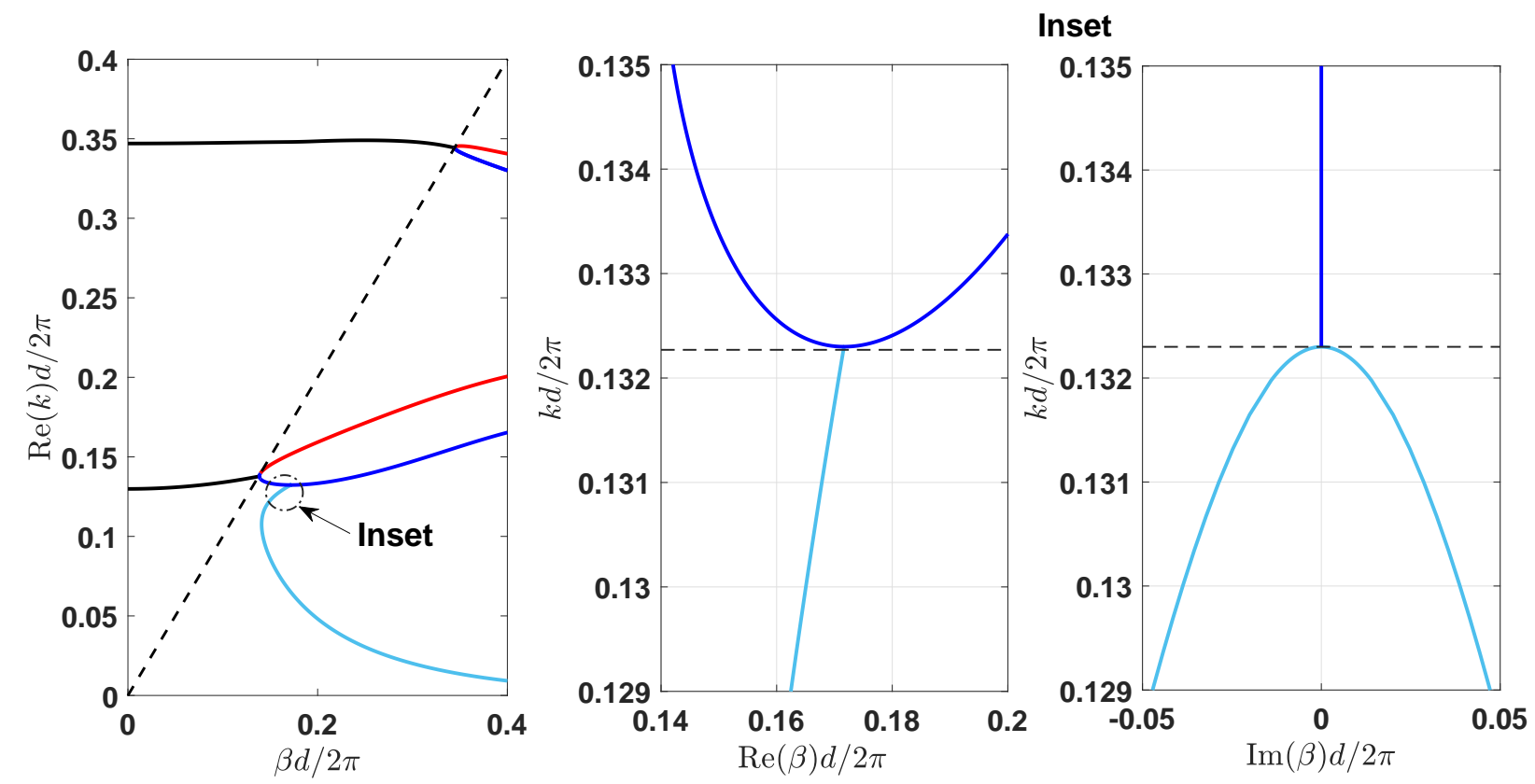

FIG. 7: Dispersion curves of two even modes for a periodic slab with $h=3 d, \varepsilon_{1}=11.56, \varepsilon_{2}=1$ and $a=0.5 d$. The guided, resonant, improper and leaky modes are shown in red, black, blue and cyan, respectively.

Light, 2nd ed. (Princeton University Press, Princeton, New Jersey, 2008).

[3] J. von Neumann and E. Wigner, "“Über merkwürdige diskrete eigenwerte," Z. Physik 50, 291-293 (1929).

[4] C. W. Hsu, B. Zhen, J. Lee, S.-L. Chau, S. G. Johnson,
J. D. Joannopoulos, and M. Soljačić, "Observation of trapped light within the radiation continuum," Nature 499, 188-191 (2013).

[5] C. W. Hsu, B. Zhen, A. D. Stone, J. D. Joannopoulos, and M. Soljačić, "Bound states in the continuum," Nat. 
Rev. Mater. 1, 16048 (2016).

[6] A. Kodigala, T. Lepetit, Q. Gu, B. Bahari, Y. Fainman, and B. Kanté, "Lasing action from photonic bound states in continuum," Nature 541, 196-199 (2017).

[7] T. Kato, Perturbation Theory for Linear Operators (Springer, Berlin, 1966).

[8] W. D. Heiss, "The physics of exceptional points," J. Phys. A: Math. Theor. 45, 444016 (2012).

[9] B. Zhen, C. W. Hsu, Y. Igarashi, L. Lu, I. Kaminer, A. Pick, S.-L. Chua, J. D. Joannopoulos, and M. Soljačić, "Spawning rings of exceptional points out of Dirac cones," Nature 525, 35 (2015).

[10] P. M. Kamiński, A. Taghizadeh, O. Breinbjerg, J. Mørk, and S. Arslanagić, "Control of exceptional points in photonic crystal slabs," Opt. Lett. 42, 2866-2869 (2017).

[11] H. Zhou, C. Peng, Y. Yoon, C. W. Hsu, K. A. Nelson, L. Fu, J. D. Joannopoulos, M. Soljačić, and B. Zhen, "Observation of bulk fermi arc and polarization half charge from paired exceptional points," Science 359, 1009-1012 (2018).

[12] A. Abdrabou and Y. Y. Lu, "Exceptional points of resonant states on a periodic slab," Phys. Rev. A 97, 063822 (2018).

[13] W. Chen, S. K. Özdemir, G. Zhao, J. Wiersig, and L. Yang, "Exceptional points enhance sensing in an optical microcavity," Nature 548, 192 (2017).

[14] H. Hodaei, A. U. Hassan, S. Wittek, H. Garcia-Gracia, R. El-Ganainy, D. N. Christodoulides, and M. Khajavikhan, "Enhanced sensitivity at higher-order exceptional points," Nature 548, 187 (2017).

[15] C. E. Rüter, K. G. Makris, R. El-Ganainy, D. N.
Christodoulides, M. Segev, and D. Kip, "Observation of parity-time symmetry in optics," Nat. Phys. 6, 192 (2010).

[16] L. Feng, Z. J. Wong, R.-M. Ma, Y. Wang, and X. Zhang, "Single-mode laser by parity-time symmetry breaking," Science 346, 972-975 (2014).

[17] B. Peng, Ş. K. Özdemir, S. Rotter, H. Yilmaz, M. Liertzer, F. Monifi, C. M. Bender, F. Nori, and L. Yang, "Loss-induced suppression and revival of lasing," Science 346, 328-332 (2014).

[18] T. Goldzak, A. A. Mailybaev, and N. Moiseyev, "Light stops at exceptional points," Phys. Rev. Lett. 120, 013901 (2018).

[19] S. Fan and J. D. Joannopoulos, "Analysis of guided resonances in photonic crystal slabs," Phys. Rev. B 65, 235112 (2002).

[20] S. Yamaguchi, A. Shimojima, and T. Hosono, "Analysis of leaky modes supported by a slab waveguide," Electron. Comm. Jpn. Pt. II 73, 20-31.

[21] G. W. Hanson and A. B. Yakovlev, "An analysis of leakywave dispersion phenomena in the vicinity of cutoff using complex frequency plane singularities," Radio Sci. 33, 803-819 (1998).

[22] J. P. Berenger, "A perfectly matched layer for the absorption of electromagnetic waves," J. Comput. Phys. 114, 185-200 (1994).

[23] W. C. Chew and W. H. Weedon, "A 3D perfectly matched medium from modified Maxwell's equations with stretched coordinates," Microw. Opt. Technol. Lett. 7, 599-604 (1994). 